\title{
Carlos Lévy et Lucia Saudelli (éd.), Présocratiques latins : Héraclite, traductions, introductions et commentaires
}

Pinelopi Skarsouli

\section{OpenEdition}

12 Journals

Édition électronique

URL : https://journals.openedition.org/philosant/709

DOI : 10.4000/philosant.709

ISSN : 2648-2789

Éditeur

Éditions Vrin

\section{Édition imprimée}

Date de publication : 1 novembre 2016

Pagination : 230-232

ISBN : 978-2-7574-1472-9

ISSN : 1634-4561

Référence électronique

Pinelopi Skarsouli, «Carlos Lévy et Lucia Saudelli (éd.), Présocratiques latins: Héraclite, traductions, introductions et commentaires », Philosophie antique [En ligne], 16 | 2016, mis en ligne le 01 novembre 2018, consulté le 02 décembre 2022. URL : http://journals.openedition.org/philosant/709; DOI : https://doi.org/10.4000/philosant.709

\section{(2) $(1 \Theta \Theta$}

Creative Commons - Attribution - Pas d'Utilisation Commerciale - Pas de Modification 4.0 International - CC BY-NC-ND 4.0

https://creativecommons.org/licenses/by-nc-nd/4.0/ 
dualists, as Plutarch implies, they need to explain why we fail to know the intelligible reality, the Forms, that account for the identity of sensible things, especially since, as the Theaetetus suggests $(185 \mathrm{~cd})$, it is the soul that perceives through the senses. Plutarch himself maintains a form of scepticism that Bonazzi terms "metaphysical scepticism". According to this version of scepticism, it is the overall human cognitive ability that is doubted given our, human, limitations (p. 101). This sense of scepticism may be found also in Alcinous, claims Bonazzi, for our ability to know is said again to be constrained by our confinement in body (Didaskalikos 155.20-36; p. 109). The reference to notions, ennoiai, that we find in both Plutarch and Alcinous, is not sufficient for winning against the challenge of scepticism. It is Plotinus who will fight scepticism by defending the possibility of man to liberate his intellect from bodily constraints. The last chapter of the book that is devoted to Plotinus focuses on this line of thought.

Bonazzi examines some important passages of Plotinus (esp. from Ennead V.5) that show clearly that for Plotinus the perception of a sensible object is an image (eidolon) and as such is fallible. The fallibility of sense perception is a consequence of the metaphysical status of the perceived object. This, however, does not mean that we are not in a position to acquire true knowledge. This can be attained by the intellect, or the non descended soul, which is capable of recognizing the intellectual reality, that is, what truly is. Bonazzi is right to claim that Plotinus is not confronting scepticism directly (p. 144) and he is also right to stress the similiarities between Plotinus view about the identity of the intellect and the intelligibles and the Peripatetic theory of intellect as we find it in Alexander of Aphrodisias (p. 146-148). But this does not justify the claim, at least not fully, that Plotinus seeks to redefine Platonism through a critical dialogue with Aristotle (p. 148). For as Bonazzi himself acknowledges (p. 148, n. 4), this dialogue with Aristotle permeates Platonism since Antiochus, if not earlier.

Bonazzi shows well, however, that late ancient Platonists are continuously engaged with scepticism; they try different tools in dealing with the sceptical challenge, such as the elements of Stoic epistemology, Plato's Forms, or the Peripatetic theory of intellect. Bonazzi's book is a very welcome addition to the literature about late ancient Platonists and indeed one that stresses the important role of the Platonist epistemological concerns in how their overall interpretation of Plato was shaped. It deserves to be studied by all scholars of late ancient philosophy.

George Karamanolis University of Vienna

Carlos Lévy et Lucia Saudelli (éd.), Présocratiques latins : Héraclite, traductions, introductions et commentaires, Paris, Les Belles Lettres, 2014 (coll. Fragments, 17), LXXII-200 p., ISBN : 978-2-251-74215-1.

Ce livre, issu du programme « Présocratiques Grecs/Présocratiques Latins » financé par l'Agence Nationale de la Recherche (ANR) et dirigé par André Laks et Carlos Lévy, est le fruit d'une tentative fort intéressante de mettre l'accent sur la question de la spécificité de la transmission et de l'utilisation latine des présocratiques. Car sans nul doute, dans le domaine des études présocratiques, les témoignages issus de la tradition latine ne se trouvent pas au même niveau que ceux provenant de la tradition grecque, le préjugé commun étant que la tradition latine ne représente qu'une information grecque traduite, plus ou moins maladroitement, en latin. lxxii

Les auteurs ont entièrement raison de souligner que cette approche se révèle trop réductrice, et tentent de réparer cette injustice en commençant par Héraclite ; ils nous 
fournissent un premier recueil, très soigné, de tous les témoignages sur le philosophe éphésien conservés en latin, avec introduction, traductions et commentaires, éclaircissant ainsi les cheminements qui ont permis la diffusion et la transmission de son œuvre, et l'apport de ces fragments latins à l'étude des sources grecques. Dans l'Héraclite latin, on retrouve aisément l'Héraclite que l'on connaît, mais sous une lumière et dans un contexte singuliers, ayant subi l'épreuve d'une langue et d'une culture autres. L'intérêt principal de l'ouvrage consiste dès lors principalement, du fait de son approche ciblée, dans le rassemblement et l'étude détaillée des textes et témoignages concernant Héraclite chez les auteurs latins, et dans la mise en évidence de leur regard propre sur un matériel déjà plus ou moins connu.

La première partie de l'introduction (p. ix-xv) décrit les caractéristiques générales de la philosophie à Rome, et plus précisément son rapport à la réception des présocratiques ; elle donne un avant-goût de la qualité globale de l'analyse, soucieuse des détails, et prenant soin d'étayer ses propos par des exemples et des références bibliographiques ; une analyse, parfois rapide, mais ne perdant jamais de vue la trame de l'ouvrage, faisant preuve de clarté et aussi de prudence quant à l'usage et à l'évaluation du matériel.

L'introduction aborde ensuite « la question doxographique » (p. xv-xxi), question centrale pour les présocratiques, d'autant plus que les auteurs nous font part de leur intention de problématiser à leur tour ce concept très discuté en histoire de la philosophie qu'est la « doxographie », en étudiant le cas particulier des auteurs latins (p. xvii). Vient ensuite l'exposé des structurations de l'histoire de la philosophie chez les auteurs de langue latine (p. xxi-xxix) ; sont abordés les exemples de Lucilius, Lucrèce, Cicéron et Sénèque, ainsi que la notion plus générale de physicus ( « La catégorie des physici », p. xxix-xxxiii).

La seconde partie de l'introduction (p. xxxiii-lxxii) se concentre sur Héraclite. Elle rappelle les principaux traits connus de la figure d'Héraclite, ainsi que ceux de sa doctrine, dans leur contexte grec et notamment par rapport aux différents courants philosophiques jusqu'à l'avènement du christianisme ; elle se concentre ensuite sur l'étude, au cas par cas, de leur réception latine à l'époque hellénistique et romaine.

Nous souhaitons réserver une mention particulière, à titre d'exemple, à un chapitre appartenant à cette partie de l'introduction, « Les citations d'Héraclite » (p. xli-xlv), assez représentatif à nos yeux de la démarche qu'adoptent les auteurs et de l'intérêt particulier de leur ouvrage concernant l'étude des textes. Ce bref chapitre nous apprend que les auteurs latins nous fournissent plus de comptes rendus indirects que de citations littérales d'Héraclite, et pose la question de ce que cela pourrait signifier quant à leurs sources, mais surtout quant à leurs intérêts et à leurs objectifs. Il souligne aussi le fait que certains de ces auteurs citent Héraclite en grec (c'est le cas par exemple d'Apulée ; le passage tiré de son œuvre, Mund. 20, 335, est analysé de près) et examine les raisons de cette pratique. D'autres auteurs enfin, de Cicéron à Tertullien, traduisent Héraclite en latin - tel Cicéron (Tusc. V, 105), qui paraphrase la première partie du fragment héraclitéen 22 B 121 DK tandis qu'il en cite littéralement la fin. Suit un autre témoignage significatif, celui de Sénèque $($ Epist. 58, 22) sur ce qu'on appelle la doctrine « des fleuves » d'Héraclite, qui est bien plus que la simple version latine du fragment héraclitéen en question (22 B 49a DK), ainsi que le texte de Columelle $(8,4)$, le cas le plus remarquable de citation en latin d'Héraclite (22 B $37 \mathrm{DK})$, un des très rares « fragments latins » d'Héraclite chez Diels-Kranz.

Après cette riche introduction, débute le recueil des témoignages proprement dit (p. 1-175). Les textes latins présentés sont, sauf indication contraire, ceux de la Collection des Universités de France. Pour chaque témoignage sont indiquées les 
références aux éditions Diels-Kranz et/ou Mouraviev d'Héraclite. Toutes les traductions sont originales.

Ce vaste ensemble de témoignages est regroupé par auteur. On décompte vingthuit entrées, de Varron à Augustin, qui se succèdent selon l'ordre chronologique, les plus fournies étant celles de Cicéron, Lucrèce, Sénèque, Apulée, Tertullien et Calcidius. Chaque entrée est précédée d'une brève et utile introduction concernant surtout l'œuvre d'où le témoignage est extrait ; le texte latin est suivi de la traduction originale, presque toujours limpide, qui en est proposée (on regrettera seulement que cette dernière ne se démarque pas typographiquement de façon plus explicite du reste du texte) et de minutieuses notes de bas de page en guise de commentaire. Enfin, chaque notice est close par une bibliographie ciblée.

La dernière partie de l'ouvrage, «Conclusions » (p. 177-179), revient non sans humour et avec la prudence habituelle des auteurs sur la qualité historique et philosophique de l'Héraclite latin. À l'évidence, la tradition latine n'est pas novatrice ; elle se réduit pour l'essentiel à quelques thèmes, stéréotypés, sans cesse repris, liés entre autres aux anecdotes concernant Héraclite et aux formules qui l'accompagnent dans toute la littérature antique. L'Héraclite latin est donc le philosophe-qui-pleure, le penseur obscur, l'inventeur du feu comme principe de toutes choses. En revanche, la filiation Héraclite-stoïcisme est beaucoup moins présente, contrairement à ce que l'on pourrait attendre. Ainsi le thème du flux semble avoir laissé les Romains indifférents à l'exception de Sénèque. Cependant, comme le soulignent à juste titre Carlos Lévy et Lucia Saudelli (p. 179), « peut-être l'Héraclite romain n'était-il ni très original ni très profond, mais le simple fait qu'il existât représentait en lui-même un fait majeur ».

Un index locorum (p. 181-190) clôt cet ouvrage remarquable. Une remarque finale des auteurs sonne comme une promesse, puisqu'elle laisse entendre que ce livre est à considérer comme le premier d'une série destinée à donner l'image la plus exacte possible de ce que représentèrent les présocratiques pour les Romains cultivés - et ce d'autant plus qu'Héraclite est le plus souvent accompagné dans les textes étudiés de mentions d'autres présocratiques. Nous avons cru comprendre que Démocrite, l'image inversée du philosophe éphésien, devrait lui succéder; nous attendons le cas échéant la suite de la collection avec impatience.

Pinelopi SKarsouli CNRS, UMR 8230, Centre Jean Pépin

Gretchen Reydams-Schils (éd.), Thinking Through Excerpts: Studies on Stobaeus, Turnhout, Brepols Publishers, 2011 (Monothéismes et Philosophie), 730 p., ISBN : 978-2-503-52976-9.

Cet ouvrage, qui contient les actes d'une conférence internationale tenue en mars 2008 à l'Université Catholique du Sacré Cœur de Milan, permet de mesurer les progrès réalisés dans la connaissance de Stobée depuis les Doxographi Graeci de Diels, sur les plans tant philologique que doxographique et thématique. L'épaisseur du volume, muni d'un index locorum et d'une bibliographie étoffée, interdit de faire autre chose, dans le cadre d'un compte-rendu, qu'indiquer brièvement les points saillants de chacune des seize contributions qu'il contient.

Contre le préjugé dont souffre traditionnellement l'ouvrage de Stobée, David Konstan («Excerpting as a Reading Practice », p. 9-22) montre que le genre littéraire de la compilation, loin d'être le signe d'une phase décadente de la civilisation, révèle au contraire la culture gréco-latine à son point culminant. Faisant suite à cette étude, celle de Denis Michael Searby («The Intertitles in Stobaeus : Condensing a Culture », 